\title{
Carcinoma Right Breast with Right Inguinal Lymph Nodes Metastasis, A Rare Case Report
}

\section{Hemant Pandey*, Sapna Manocha, GK Jadhav and Divya Piyushi}

Department of Radiation Oncology, Indraprastha Apollo Hospital, New Delhi, India

*Corresponding Author: Hemant Pandey, Department of Radiation Oncology, Indraprastha Apollo Hospital, New Delhi, India.
Received: September 13, 2021

Published: September 18, 2021

(C) All rights are reserved by Hemant Pandey., et al.

\begin{abstract}
Most common lymphatic spread of carcinoma breast is ipsilateral axiilary lymph nodes, internal mammary lymph nodes and supraclavicular lymph nodes. Inguinal lymph nodes involvement in carcinoma breast is quite uncommon. Surgery and irradiation for breast cancer may interfere with conventional pathways of spread, leading to bizarre patterns of dissemination through lymphatics or through hematogenous route. Other possible reasons could be occurrence of primary breast cancer in accessory breast tissue retained in the vulva following involution of milk line. We describe a case of right breast carcinoma who developed right ingquinql lymph node mets, brain mets after one month of neoadjuvant chemotherapy and mastectomy.
\end{abstract}

Keywords: Inguinal Lymph Node Mets; Breast carcinoma

\section{Introduction}

The lymphatic spread of carcinoma breast to the regional lymph nodes (axillary, supraclavicular and internal mammary lymph node chain) and hematogenous spread to the bone, liver, lung and breast is well known. However, involvement of distant nodal regions e.g. inguinal lymph nodes is extremely uncommon. These lymphatic pathways have been reported to be altered after the treatments such as axillary lymph node dissection (ALND) and/or radiation [1-4]. The study of preoperative lymphoscintigraphy for the patients with ipsilateral breast cancer recurrences showed that aberrant lymphatic drainages were relatively common in patients with ALND and/or RT [2].We present a case of estrogen receptor negative, Her2Neu inconclusive patient who developed right inguinal node metastases along with brain mets after 1 month of mastectomy post neoadjuvant chemotherapy.
Case

A 41 year old, non-diabetic, non-hypertensive female who is a known case of Psoriasis and Impetigo, presented with a lump in her right breast. On further evaluation she was diagnosed with Invasive ductal Carcinoma Right breast.

CECT chest revealed a heterogeneously enhancing 2.1 x 2 x 1.5 $\mathrm{cm}$ lesion in the retro-areolar region of right breast along with multiple right axillary lymph nodes. She received 8 cycle neo-adjuvant chemotherapy $(\mathrm{AC}+\mathrm{T})$ followed by Right breast modified mastectomy (MRM).

HPR s/o Invasive breast carcinoma, NST, grade-III, margins free, LNs 3/5 positive with extra-nodal extension. IHC: ER/PR - negative, Her2 Neu inconclusive.

After one month of MRM she presented with complaints of dysphagia to solid which progressed to liquids and associated with 
nausea, vomiting, headache, gait instability and generalized weakness. She also complaints of on and off vertigo. She was also diagnosed with COVID 19 and treated according to mild COVID protocol.

A CECT head and chest was performed which revealed metastatic lesions in right parieto-occipital region and medulla with peri-lesional edema. She was started on anti-edema measures and Ryle's tube feed. She then presented to our institute for further management.

A PET-CT scan was done which revealed metastatic lesions in pons of size $2 \times 1.9 \mathrm{~cm}$ and another centimeter size lesion in the cerebellum, FDG avid level V cervical lymph nodes, and FDG avid enlarged right external and inguinal lymph nodes seen largest short axis diameter $1.5 \mathrm{~cm}$ in right inguinal region.

FNAC was done from the inguinal LN which revealed invasive ductal carcinoma. Subsequently, she received adjuvant radiation with 40 Gy in 15 fractions to right chest wall, whole brain radiotherapy (WBRT) with 30 Gy in 10 fractions along with simultaneous integrated boost to brain mets with 40 Gy in 10 fractions. She also received 45 Gy in 15 fractions to the gross inguinal lymph nodes with $9 \mathrm{MeV}$ electron beam.

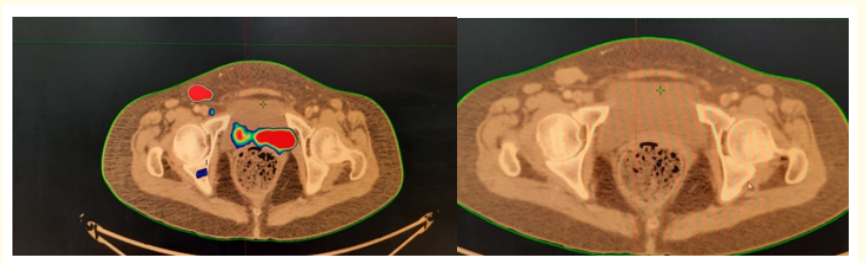

(a)

(b)

Image 1: a. Shows FDG avid right inguinal LNs metastasis, b. (CECT) shows enlarged right inguinal LNs.

\section{Discussion}

Most common route of spread of carcinoma breast is through hematogenous and lymphatic pathways. Metastatic breast cancer can involves almost all organs of the body. Lymphatic route of spread involves regional lymph nodes while hematogenous route of spread involves in distant metastasis $[5,6]$. The regional spread can occur at the ipsilateral axillary, supraclavicular and sometimes at the ipsilateral internal mammary chain.

Inguinal lymph nodes involvement is extremely rare in case of carcinoma breast and it is believed that this lymph nodes can sometime involves after occlusion and disruption of lymphatic drainage following axillary dissection or irradiation $[7,8]$. Non-regional lymph node involvement by breast cancer has been described earlier in mediastinum, para-aortic and pelvic lymph nodes. Metastasis of breast cancer to inguinal lymph nodes is extremely rare [5,9]. The first such report by Baba et al. described a patient with inguinal node and breast lump at presentation [6].

Retrograde embolization was suggested as another probable pathogenesis. It has been postulated that in the presence of altered lymphatic pathways after surgery or irradiation, such involvement should be regarded as regional recurrence and considered for radical therapy [10]. Presence of ectopic breast tissue in the vulva with inguinal nodal involvement could be the other possible etiology [9].

A lymphoscintigraphy study on 23 patients of operable breast cancer before and after therapy that included axillary dissection, irradiation, or both, determined that the incidence of contralateral axillary lymph node spread increased from $1 / 23$ at baseline to 6/23 after therapy [11].

In the present case as well as the available literature, altered lymphatic pathways could have been responsible for the inguinal involvement, but without doubt, inguinal involvement was associated with early systemic progression since the patient also had two brain mets. Selected subset of metastatic patients should be approached with curative, not palliative, intent.

Therapeutic developments, such as the introduction of new cytotoxic agents (Taxanes, Vinorelbine, Capecitabine, Gemcitabine, etc), third generation aromatase inhibitors and fulvestrant, Targated therapies (Trastuzumab, Lapatinib, and Bevacizumab), Immunotherapy and cell cycle inhibitors have resulted in constant improvements in treatment efficacy and consequently in metastatic outcome [12].

\section{Conclusion}

The biological behavior of breast cancer is well known. Although the abnormal involvement of distant lymph nodes e. g. inguinal lymph nodes in case of carcinoma breast is rare and it might

Citation: Hemant Pandey., et al. "Carcinoma Right Breast with Right Inguinal Lymph Nodes Metastasis, A Rare Case Report". Acta Scientific Cancer Biology 5.10 (2021): 19-21. 
happens because of aberrant lymphatic architecture due to surgery or radiation. The early systemic involvement of disease cannot be ruled out hematogenous spread to the lymph nodes. As the number of patients with unusual site involvement is increasing, the clinician should be extra cautious when coming across unusual complaints and findings.

\section{Conflict of Interest}

No.

\section{Bibliography}

1. Kaur P., et al. "Sentinel lymph node biopsy in patients with previous ipsilateral complete axillary lymph node dissection". Annals of Surgery Oncology 18 (2011): 727-732.

2. Sato A., et al. "Altered lymphatic drainage patterns in re-operative sentinel lymph node biopsy for ipsilateral breast tumor recurrence". Radiation Oncology 14 (2019): 159-165.

3. Renata M., et al. "Scintigraphic visualization of an epigastric sentinel node in recurrent breast cancer after lumpectomy and postoperative radiation therapy". Clinical Nuclear Medicine 31 (2006): 207-208.

4. Richard R., et al. "Unexpected lymphatic drainage of the treated breast". Clinical Nuclear Medicine 44 (2019): 732-734.

5. Shikha Goyal., et al. "Case report: Breast cancer with inguinal node recurrence". Journal of the Egyptian National Cancer Institute 27 (2015): 41-43.

6. Baba M., et al. "A case of breast cancer diagnosed by inguinal lymph node metastasis". Breast Cancer 7 (2000): 173-175.

7. Kilciksiz S., et al. "Isolated inguinal lymph node metastasis from breast carcinoma -case report and review of the literature". J BUON 11 (2006): 229-232.

8. Wellner R., et al. "Altered lymphatic drainage after breast-conserving surgery and axillary node dissection: Local recurrence with contralateral intramammary nodal metastases". Clinical Breast Cancer 7 (2007): 486-488.

9. Sood A., et al. "Alternative lymphatic pathway after previous axillary node dissection in recurrent/primary breast cancer". Clinical Nuclear Medicine 29 (2004): 698-702.
10. Jebbin NJ and Adotey JM. "Metastatic carcinoma of the breast with inguinal lymph node involvement: a report of two cases". Nigerian Journal of Clinical Practice 11 (2008): 383-385.

11. Perre CI., et al. "Altered lymphatic drainage after lymphadenectomy or radiotherapy of the axilla in patients with breast cancer". British Journal of Surgery 83 (1996): 1258.

12. William Wood., et al. "International Guidelines for Management of Metastatic Breast Cancer: Can Metastatic Breast Cancer Be Cured?" Journal of the National Cancer Institute 102 (2010): 456-463.

\section{Volume 5 Issue 10 October 2021}

(C) All rights are reserved by Hemant Pandey., et al. 